\title{
Differentiated Thyroid Gland Carcinoma 55 Years and Older AJCC v8 Stage
}

National Cancer Institute

\section{Source}

National Cancer Institute. Differentiated Thyroid Gland Carcinoma 55 Years and Older A/CC v8 Stage. NCI Thesaurus. Code C140964.

A differentiated thyroid gland carcinoma stage for patients 55 years and older defined according to the AJCC 8th edition criteria. 